\title{
La dinámica de las ciudades portuarias regionales. Un campo de investigación transdisciplinar
}

Miguel Ángel De Marco (h)

Instituto de Estudios Históricos, Económicos, Sociales e Internacionales, Consejo Nacional de Investigaciones Científicas y Técnicas, Argentina.

https://orcid.org/000o-0002-6259-0224

Recibido: 29 de marzo de 2019. Aceptado: 30 de junio de 2020.

\begin{abstract}
Resumen
La renovación de las distintas escuelas y propuestas historiográficas, en especial aquellas que han sido permeables en las últimas décadas a los enfoques interdisciplinares aportados desde la economía, el urbanismo, la geografía, y la logística, ha contribuido a la apertura de un nuevo campo para la comprensión de la incidencia de los puertos en las transformaciones territoriales: el estudio de la dinámica histórica de la relación entre ciudades puertos y regiones, y su incidencia en las características del desarrollo existente en una región; temática que ha requerido de una investigación transdisciplinar. En el presente artículo se compartirá el proceso de construcción teórica que permitió definir este nuevo campo, la metodología empleada, y sus desafíos. Asimismo, se reseñará la conformación del Núcleo de las Ciudades Portuarias Regionales IDEHESI-Conicet, las actividades realizadas y las experiencias derivadas de la misma.
\end{abstract}

Palabras Clave: Puertos. Desarrollo. Región. Historia.

\section{The dynamics of regional port cities. A transdisciplinary research field}

\begin{abstract}
In the last decades, a new field has been opened por the comprehension of the impact of ports in territorial transformations, due to the renewal of different historiographic schools and approaches, especially those that have been permeable to interdisciplinary perspectives coming from economy, urbanism, geography, and logistics: the study of the historical dynamics of the relationship between port cities and regions, and its effects in the characteristics of the existing development in a region; subject that by its complexity has required a transdisciplinary investigation. This paper presents the theoretical construction process that allowed to define this new field, the methodology used, and its challenges. Likewise, the conformation of
\end{abstract}


the Núcleo de las Ciudades Portuarias Regionales (Core of the Regional Port Cities) IDEHESI-CONICET, the activities carried out and the experiences derived from it, will be outlined.

Keywords: Ports. Development. Region. History.

Palavras-chave: Portos. Desenvolvimento. Região. História.

\section{Interpelaciones contemporáneas}

Treinta años después de la aceleración del proceso de mundialización económica y de la intensificación de los avances tecnológicos se acentúan tensiones entre la actividad portuaria, y las ciudades donde se desenvuelven. Avanzar en un sistema de gobernanza para el desarrollo sustentable de las ciudades portuarias es un propósito reconocido por los gobiernos y la sociedad civil de los países más preocupados por atender y resolver el juego de demandas globales y locales.

La investigación de la dinámica histórica de las ciudades portuarias se erigiría en una herramienta válida al diálogo entre disciplinas sobre esta temática.

Diversos encuentros científicos/académicos internacionales de cultura portuaria, entre los que se encuentran los organizados por IDEHESI-CONICET señalan la rápida disminución observada en las últimas décadas del acervo patrimonial de los puertos, incluyendo documentación de las instituciones del Estado encargadas del área, adquiriendo una situación crítica en el caso argentino.

Detectar a los actores, saberes y producciones tangibles intervinientes en la interacción puerto-ciudad-región, en sus distintos niveles, y tiempos históricos, podrían contribuir a la identificación, localización, preservación y digitalización para la conservación y estudio del patrimonio portuario en riesgo de desaparición, al mismo tiempo que progresar en la comprensión de la dinámica de relación que ha incidido en las etapas gobernanzas.

El presente trabajo se enfoca en describir la evolución historiográfica que ha permitido fundamentar tal hipótesis como parte de la ejecución de un proyecto de cooperación internacional Francia y Argentina, y un PICT de la Agencia Nacional de Promoción Científica y Tecnológica. ${ }^{1}$

La comprensión de la estructura funcional de las ciudades portuarias y sus etapas de evolución adquirió singular interés en la segunda mitad del siglo XX, contemporáneamente con la imposición de nuevos patrones en la operatoria del comercio internacional naval que condujeron a la relocalización y a la refuncionalización de las que, localizadas en el centro de la ciudad, quedaron en desuso. Esta inquietud se tornó cada vez más apremiante cuando hacia la década del 80 y 90, se aceleraron las innovaciones tecnológicas y las reglas aperturistas del comercio internacional requirieron apresurar las transformaciones en la morfología de los frentes portuarios (Tobal Conesa, 1997). Programas y proyectos urbanísticos y medioambientales dedicados al mejoramiento de la calidad de vida de las ciudades, patrocinados por la Unión Europea, distintos

1 El Proyecto Ecosud 2018 se denomina "Las humanidades digitales aplicadas al estudio comparado del impacto urbano y regional de la modernización tecnológica de los puertos de ultramar de Francia y Argentina”, entre el Centro Francois Vieté de la Universidad de Bretaña Occidental (Francia) y el Idehesi Conicet, dirección Serge Gerlatti y Miguel Ángel De Marco (h); y el PICT 2018 “Las humanidades digitales aplicadas al estudio comparado del impacto urbano y regional de la modernización tecnológica de los puertos de ultramar de Francia y Argentina”, dirección de Miguel Ángel De Marco (h). 
organismos internacionales y las propias autoridades portuarias, financiaron la investigación de las secuencias de interfaces entre los puertos y las ciudades. Organismos públicos y privados, por otra parte, recurrieron a identificar a su urbe como una "ciudad puerto", enfatizando la adhesión a políticas de inserción internacional. ${ }^{2}$

Brian Stewart Hoyle, investigador de la Universidad de Southampton, Gran Bretaña, conformó un equipo interdisciplinario que estableció directrices comunes que permitieran estudiar y comparar las ciudades portuarias entre sí (Hoyle, 1992). A su vez el referido científico, basado en los estudios de "Teoría General de los Sistemas", presentada en 1968 por Ludwig von Bertalanffy despertó la atención de geógrafos de la Universidad de Barcelona, donde se creó la cátedra de Geografía Portuaria ${ }^{3}$, y en la Asociación Internacional Villés et Ports (AIVP), entre otros ámbitos académicos.

En 1985 Hoyle y D. Hilling, dieron a conocer principios teóricos que sirvieron de referencia a los geógrafos portuarios proponiendo los temas que habría de tratar esta especialidad, dividiéndolos en cuatro grandes grupos: teóricos, comprendían el análisis de la interdependencia entre cuidad-puerto-industria; interdisciplinarios, el sistema de transporte-puerto-desarrollo-políticas portuarias; espaciales, local-regional-área de influencia comercial-internacional; y temporales, velocidad-servicios del envío de mercadería-procesos de selección de puertos. Temas que a su vez podrían ser estudiados en escala municipal, regional, nacional e internacional y en contextos según la evolución de cada economía en cuanto a su desarrollo: avanzado, mediano o menor (Hoyle y Hilling, 1985).

La Asociación Internacional Villes et Ports, junto a la Organización de las Naciones Unidas y la Asociación Internacional de Puertos decidieron efectuar en 1996 un informe conjunto para una mejor comprensión de la problemática en el tiempo presente y adoptar decisiones conducentes a un desarrollo sustentable. Apoyándose en los estudios de Frank Broeze y B. Hoyle, el documento reconoció un distanciamiento físico, social y cultural entre "los puertos y las ciudades" a medida que la sociedad industrial fue haciendo inviable la coexistencia de grandes complejos portuarios en los conglomerados urbanos.

\section{La renovación historiográfica}

La Segunda Guerra Mundial y la Guerra Fría fomentaron el interés por la historia marítima en relación con la geopolítica y geoestrategia naval, y los enclaves portuarios. Hasta entonces se habían editado abundantes trabajos descriptivos y empíricos sobre el pasado y presente de los puertos, los que quedaban habitualmente subsumidos en la historia marítima. Sería la Escuela de los Annales las que sustrayéndose de dichas inquietudes dará cabida a una amplitud de trabajos que estudiaron a los puertos en relación con ciudades y rutas. Uno de sus representantes más encumbrados, Fernand

2 Precisamente tras la intención de favorecer "la simbiosis ciudad y puerto" se creó en 1912 la institución decana de estas características, la Asociación Americana de Puertos (AAPA), en Estados Unidos de Norteamérica. En 1988, se constituyó una estructura de enlace entre ciudades y puertos: La Asociación Internacional Villes et Ports; en 1991 nació la Agrupación de Ciudades Puertos de Chile y en 1994, la Red de Ciudades Puerto del Cono Sur de América comprometiéndose a "abordar y solucionar la problemática propia de la identidad y proyección del concepto ciudad puerto vinculado al desarrollo comunal, regional, nacional e internacional”. En 2001 surgió la RETE, una red internacional de ciudades portuarias y puertos, destinada, entre otros aspectos a mejorar la integración de los puertos con su entorno territorial, económico, social, cultural y medioambiental.

3 Fue su primera titular la doctora Rosa Castejón, del Departamento de Geografía Física y Análisis Geográfico Regional de dicha Universidad y asesora de AIVP. 
Braudel, produjo una obra bisagra: El Mediterráneo y el mundo mediterráneo en la época de Felipe II, de 1949, la que implicó un salto cualitativo en los estudios de la historia de la actividad portuaria por su reflexión teórica y metodológica. Introdujo consideraciones acerca de las acciones ejercidas por las ciudades puertos en el devenir histórico: como la de atraer, expulsar, y señalar cambios, y las contextualizó dentro de la dinámica del capitalismo. Destacó la persistencia de un mismo patrón: la conformación de "economías mundo" -lideradas por dos ciudades puertos dominantes-, divididas en zonas: un corazón; las zonas intermedias, pivotes del poder central; y las marginales. Era precisamente del escalonamiento entre las ciudades puertos dominantes, las zonas intermedias, y las periféricas donde el capitalismo obtenía sus mayores réditos. Por eso, como mediadoras de la distancia, favorecían a los que negociaban la comunicación, desde el viajante al consignatario (Braudel, 1977).

En las últimas décadas del siglo XX los crecientes trabajos presentados en coloquios, simposios y congresos históricos sobre los puertos y las ciudades tomaron giros diversos dependiendo del énfasis teórico y metodológico elegido por los investigadores. La mayoría de ellos fue adscrita a la historia local, económica, regional, y social, en ese orden de desarrollo.

La historiografía anglosajona en la temática de ciudades puertos también adoptó como referencia las obras de los mencionados Broeze y Hoyle, y también las de David Pinder y Jacob Price. Este último investigador definió en 1974 la relación entre la estructura socio-profesional de los puertos y la organización económica de los mismos. Estos autores inspiraron la realización de simposios e investigaciones comparadas sobre las ciudades portuarias de los cinco continentes, dando paso a trabajos publicados por investigadores de impacto como Franklin W. Knight, Peggy K. Liss, Patrick O'Flanaggan, Alejandro de la Fuente, Sheryllynne Haggerty y Anthony Webster. Para la Argentina, ésta corriente estuvo en parte representada por los trabajos que sobre el puerto de Buenos Aires realizara Susan Socolow.

Entre las obras insoslayables que ha dado la historiografía hispanista, entre los estudiosos de las ciudades portuarias, en especial para los americanistas, se encuentra Sevilla y el Atlántico, 1504-1650, de Pierre Chaunu, editada por primera vez en doce tomos, en París en 1955.

Poco después de la expansión de los enfoques promovidos desde la geografía y el urbanismo, alentados como se dijo por la relocalización de las instalaciones portuarias, comenzaron a obtener mayor visibilidad trabajos teóricos de especialistas españoles que propusieron estudiar metodológicamente el devenir de las ciudades portuarias. Agustín Guimerá Ravina y Fernando Monge, siguiendo el camino trazado por Frank Broeze, en su obra sobre los puertos de Asia, y el prolífico investigador de los puertos españoles Carlos Martínez Shaw, instaron a una nueva visión de las ciudades puerto que incluyera a "los protagonistas de las ciudades portuarias, la propia gente", (Guimerá Ravina-Monge, 1999: 3-20) haciendo uso de las herramientas de la historia social, la historia de la cultura y la antropología. En la mayoría de las investigaciones predominaban valoraciones parciales de los puertos: los procesos de modernización tecnológica, la función de las elites locales y foráneas, el comercio exterior, la actividad mercantil y aduanera, entre otros aspectos. "Unas veces los trabajos se han limitado a un marco regional, cuando no local. Otras han enfatizado determinados procesos. Además, han priorizado el análisis de un sistema portuario concreto en detrimento de una visión general. Pero lo más relevante es que la mayoría de los autores hablan del puerto o la ciudad, pero no contemplan la ciudad portuaria como ente único. Las excepciones a esta tónica general son los trabajos de geografía urbana y del transporte. Por otra parte, en la bibliografía portuaria mundial han primado excesivamente las visiones de la historia económica y la geografía, centrándose en las funciones ejercidas en los puertos 
en los procesos de intercambio, así como la repercusión espacial de estos fenómenos. Además, alude a la ciudad y al puerto, pero pocas veces observa ambas cosas a la luz de un entramado común, el de la ciudad portuaria”. (Guimerá Ravina, 2006:210)

El aporte de Guimerá Ravina y Monge fue observar a la ciudad portuaria desde lo sociocultural, la identificación del desarrollo de una estructura social con el surgimiento de nuevas posibilidades y por ende de una dinámica específica de acuerdo a las necesidades del puerto y a las características de los productos comercializados. (Guimerá Ravina, 2003). Monge (2000) priorizó una visión antropológica de los estudios portuarios en la cual incluyó al análisis los sujetos individuales y propuso entender al puerto como una comunidad humana.

En 2008, a través de un estudio comparado e interdisciplinario de puertos españoles en el siglo XIX y principios del siglo XX, un conjunto de investigadores comprobó la simultaneidad de procesos económicos, culturales y sociales que se daban en torno de las ciudades portuarias en su carácter de nodos articuladores de los mercados en distintas escalas: internacional, nacional, regional, y local. En la actualidad el investigador canario Miguel Suárez Bosa se constituye en una referencia obligada. Junto a su equipo, encuadró su aporte "en la historia atlántica" y la metodología de la Escuela de Southampton "desde una visión diacrónica de la geografía histórica", expuesta por Hoyle en 1997 y el enfoque locacional seguido por Cristòfol Tobal Conesa. Validan la utilización del término ciudad puerto siguiendo a Hole, Pinder, Guimerá Ravina, y Jesús Mirás Araujo, este último abocado a la historia del tráfico mercantil por los puertos españoles del siglo XX. (Suárez Bosa, 2011:74).

La principal red internacional de historiadores en la temática, y que convoca a investigadores de distintas generaciones y países, es el Grupo La gobernanza de los puertos Atlánticos, siglos XIV-XXVI, creado en Oporto, Portugal, en 2012, con sede en la Universidad Nacional a Distancia de España, en Madrid. La temática de sus coloquios explicita la apertura a una variedad de enfoques: Redes portuarias (2012), Políticas y estructuras (2013), Dinámicas económicas (2014), Las ciudades portuarias y su universo cultural (2015), Puertos nuevos, puertos pioneros (2016) y Puertos y Desarrollo (2017).

La Argentina presenta una abundante producción historiográfica sobre el pasado portuario, naval, y urbano, los puertos en el federalismo y el desarrollo argentino, y más recientemente los puertos en clave interdisciplinar, a la luz de la renovación historiográfica operada a partir de las últimas décadas del siglo XX. En lo que respecta a este último punto se destaca la publicación, en 1992 del libro Estado, capital extranjero y sistema portuario argentino, de Silvia B. Lazzaro, en la que se ofreció una singular visión de conjunto, de la que hasta entonces se carecía, sobre el sistema portuario del período agroexportador. A principios de este siglo pueden señalarse las líneas de investigación abiertas por Fernando Jumar (en materia de puertos y flujos comerciales rioplatenses, siglos XVII y XVIII) de la Universidad Nacional de La Plata, formado con Bernard Lepetit (Escuela de Annales); José Mateo Oviedo (Puertos y trabajadores del mar), de la Universidad Nacional de Mar del Plata y fundador del Grupo de Estudios Sociales Marítimos (GESmar), quien siguiendo las orientaciones de Juan Carlos Garavaglia, Jorge Gelman y Raúl Fradkin, trabajó junto a Joan-Lluis Alegret y Josep Fontana. En el 2008, Jumar y Mateo, junto a Ana Ferreyra, Marta Palomares, Emir Reitano, y Marcelo Weissel, decidieron realizar encuentros anuales de investigadores interesados en la temática de los puertos, y así surgió la Red de Estudios Portuarios (REDEP), la que, a partir de entonces contribuyó al diálogo de distintas perspectivas disciplinares, y con intereses circunscriptos en la evolución del puerto. Dado lo anterior, el interés de esta investigación pasó por comprender al puerto de Rosario en su dinámica de relación con el proceso político, la construcción de imaginarios y discursos, la formación de recursos universitarios, el desarrollo santafesino y la puesta en valor del 
patrimonio portuario. Esto ocurría en un momento en que, por primera vez en su historia, el puerto local, pasaba a ser administrado por la provincia de Santa Fe, con intervención del municipio de Rosario y representantes de fuerzas vivas, a través de un Ente administrador que empezó a operar mediante una reconversión de la zona portuaria central. En este contexto se publicaron dos libros sobre la historia del puerto de Rosario, aunque ceñidos a un enfoque político institucional. Sería recién en el libro Historias en ciudades puertos (2009), a manera exploratoria, alentado por el marco institucional de una Unidad Ejecutora en Red de Conicet, de perfil interdisciplinar/ de conformación interdisciplinar como el Instituto de Estudios Históricos, Económicos Sociales e Internacionales (IDEHESI), se elaboró una aproximación metodológica en similar orientación a la trazada por Guimerá Ravina y Fernando Monge, la que fue profundizada en el libro Ciudad Puerto, universidad y desarrollo regional (2013), y en las investigaciones posteriores.

\section{Enclave portuario, ciudad con puerto, ciudad puerto, complejo de ciudades portuarias}

Las ciudades con puerto encierran una dimensión regional de dos unidades imposible de eludir: a ella convergieron elementos del hinterland (área de influencia interior de un puerto, local y regional) y foreland (área de conexiones de un puerto a través de las rutas marítimas tanto de importación como de exportación) que a lo largo de la historia le otorgaron una capacidad generadora de procesos de cambio y modernización. El término "ciudad puerto" comprendería el estudio de dos realidades no definitivamente integradas, referirse a "ciudad portuaria" implicaría el análisis de una situación consumada, simbiótica. (Guimerá Ravina, 2006:210).

El relevamiento de los autores citados en artículo, acerca de la evolución histórica de las principales ciudades portuarias de ultramar, permitiría tipificar etapas. Ellas no guardan entre sí necesariamente una correlación consecutiva y depende del contexto histórico particular: 1) Enclave portuario; 2) Enclave portuario y conglomerado poblacional; 3) Puerto con Ciudad; 4) Ciudad Puerto: dos realidades que interactúan con distintos grados de integración: baja, media, y alta; 5) Ciudades Portuarias: Situación simbiótica consumada, en aspectos económicos, políticos, sociales y culturales. El puerto puede haber sido en un momento la razón de ser de la ciudad. 6) Ciudad Portuaria Regional (en adelante $\mathrm{CPR}$ ): tres realidades con diversos grados de interacción entre sí y con el hinterland y el foreland, según la disposición de las áreas de interfaces, las que también permitiría evaluar el desarrollo del conjunto.

Siguiendo a referentes de la historiografía regional argentina, -que a su vez han recogido enfoques heredados de nuevas vertientes teóricas de la geografía humana-, la ciudad portuaria es en sí, el nodo de una "región funcional" y por eso mismo permite "aprehender en toda su riqueza el juego dialéctico entre condicionamientos y prácticas" (Areces, 1999:58) y permite observar en un recorte espacial

la expresión de la relación hombre espacio: las modalidades productivas y de intercambio; y los sujetos sociales involucrados, percibiendo de esa manera la supervivencia de una compleja red de relaciones económicas y socioculturales vigentes, detectando los elementos que facilitaron la perdurabilidad de tales contactos (Bandieri, 2005:96).

Asimismo, las CPR, intervienen en la dinámica de acumulación de poder, y, por ende en el tipo de desarrollo: horizontal y/o asimétrico, según la asignación de los beneficios. A tal fin, García y Roffman (2013) se sirven de nodos y subnodos, con los que configuran 
en espacios, territorios, ritmos, cosmovisiones, que las constituyen, identifican y generan tensiones, y con los que fundamentalmente reproducen un modelo para "garantizar su subsistencia en una cadena de relación, y con ello las acciones individuales y colectivas... En la medida que los sujetos se reconocen o definen en relación con el espacio, éste se convierte en una coordenada identitaria" (Buccarelli, 2009:9).

Por otra parte, la relación entre uno o más puertos en un espacio y tiempo determinado, implica una escala de análisis específica. Fernando Jumar estudió lo que él define como "complejo portuario rioplatense durante el Antiguo Régimen", conformado fundamentalmente por Buenos Aires y Montevideo, un espacio integrado, "complementario", al menos hasta los sucesos de Mayo de 1810 cuando el proceso revolucionario creó mercados regionales particulares y los espacios debieron reconfigurar sus relaciones, dando lugar a bloques y modelos de larga duración (Jumar, 2012). Cincuenta años más adelante se puede observar la dinámica de las CPR envuelta en la puja de los capitales ingleses y franceses por obtener la exclusividad de áreas de influencias portuarias en la Argentina agroexportadora (Regalsky, 1999). Los procesos históricos configuran complejos portuarios quienes a su vez se convierten en determinantes de dichos procesos.

La comprensión de las CPR como configuradora de los territorios no se limita a la competencia por las áreas de control de rutas y plazas comerciales, va "más allá del mercado" (Gallego Martínez, 2014), observando aspectos como la generación de conocimiento propio, crítico y complementario. De esta manera se podrá comprender la verdadera dimensión relacional de los hinterland que supera los límites usualmente conocidos. Por ejemplo, aquellos espacios y fronteras marítimas establecidas entre las elites portuarias y corporaciones mercantiles de distintas ciudades portuarias (Kraselsky y Pereyra, 2012).

\section{La ciudad portuaria regional, su dinámica}

Entender al puerto de Rosario en su carácter institucional, y como tal generador de reglas de comportamiento, procesos de sociabilización, creencias culturales, y formas de transacciones, sometido a una multiplicidad de secuencias y variables, nos llevó a reconocer la existencia de una dinámica que intervenía en su evolución e impactaba sobre su presente. $\mathrm{Al}$ igual que las instituciones que al expandirse crean complejos institucionales, los puertos extendieron su radio de acción a lo largo de su existencia experimentando interacciones, retroacciones, e inter-retroacciones "por sî" (Tobal, C. 2000), según el tipo de secuencia de interfaces temporarias, y espaciales (Broeze, 1997).

A partir de la identificación del puerto como "institución”, principal ordenadora del sistema puerto-ciudad-región (elementos de interacción unidos por la afectación mutua), refiriéndonos al caso rosarino en la segunda mitad del siglo XIX y la primera mitad del siglo XX, ponderamos su primacía entre las partes, lo que era indispensable para avanzar en el entendimiento de la dinámica regional.

El orden jerárquico de cada sistema establece su dinámica, según su capacidad de mediación como organizador (von Bertalanffy, 1968). Determinar por lo tanto la posición de la institución puerto de Rosario en dicho orden en relación con las instituciones de desarrollo de la ciudad, la región, la provincia y la nación, se presentaban sugerentes para un nuevo enfoque del pasado local, comprendiendo las estructuras y las conductas de Rosario como ciudad portuaria regional.

Desde la geografía y el urbanismo también se entiende a la ciudad puerto como un nodo de comunicación entre tierra y mar con redes que ejercen una fuerte influencia en la 
ordenación del territorio, incluyendo a la región periférica, sin que eso implique una correlación automática entre el desarrollo portuario con el desarrollo de la ciudad y la región, como se estaría poniendo de evidencia en la creciente globalización. Estudios más recientes ponen en tela de juicio que "la simbiosis" entre puerto, ciudad y región pueda darse ante las transformaciones portuarias y urbanas contemporáneas (Ducruet, 2011). La Nueva Geografía Económica promueve la realización de análisis críticos que incluyan la comprensión de los factores que fomentan el crecimiento de los puertos y su declive, estudiando caso por caso su capacidad de integración territorial y con la escala urbana y regional (Ducruet-Notteboom-De Langen, 2010).

Ducruet reflexionaba, en un trabajo publicado en 2010, que desde la geografía económica y humana se carecía de "una definición consistente de la región portuaria", por ser un concepto polifacético abarcador de distintas realidades como la zona económica del puerto, la zona logística conectada al puerto, y la zona externa entre puertos. Sin embargo, estos estudios podrían ayudar a comprender los desafíos regionales al disponer de una mejor posición y rango que el del puerto y la ciudad: "Los puertos no son entidades aisladas que se conectan a cadenas de valor virtuales. Forman parte de una economía regional, y la evolución de la economía regional afecta fuertemente el desempeño del puerto" (Ducruet, 2010:15). Este científico, junto a sus colegas Sylvain Cuyala y Ali El Hosni, gracias a un proyecto financiado por la Unión Europea llevó adelante una investigación que recurrió a una perspectiva histórica para estudiar las interdependencias a largo plazo entre los flujos mundiales de transporte marítimo y el desarrollo urbano entre 1890 y 2010 . Allí se concluye que "la prominencia de las ciudades demográficamente más grandes en la red marítima está muy ligada a su capacidad para manejar múltiples tipos de flujos, para conectarse durante más tiempo, y ejercer efectos de sinergia en la cima de la jerarquía" (Ducruet, Cuyala, El Hosni, 2018).

La Geografía del Transporte entiende a la regionalización portuaria como la cuarta parte del desarrollo portuario precedido por las etapas de establecimiento, expansión y especialización, y en la que se establecen las conexiones terrestres más extensas entre el puerto y su interior (Notteboom, 2005).

El puerto, como institución real, histórica, surgida para satisfacer necesidades sociales (en la mayoría de los casos económicas), es mucho más que una actividad económicaempresarial, aunque hay elementos de la dinámica económica y de las empresas que pueden contribuir a su conocimiento, al igual que los estudios de la dinámica del transporte marítimo y fluvial, o del comercio internacional por vía acuática. Lo mismo puede aplicarse en el caso de la ciudad y la región, que poseen sus propias dinámicas. Es entonces que surge la necesidad de precisar que nuestro interés principal pasa por el análisis de la dinámica del sistema CPR, el que por otro lado no puede ser comprendido en su interdependencia con otros sistemas y jerarquías, y menos aún si no se profundiza en aquello que da cohesión y equilibrio a los sistemas: su identidad y el flujo de energía comunicacional que a través de una dinámica de retroalimentación y autoorganización se sostienen en el tiempo (Bonil et al., 2004). Es precisamente esta dinámica que atañe a lo micro, a lo local, a lo regional, y lo internacional, un ámbito para analizar los procesos de la construcción de poder y conflictividad social (Fernández, 2008) en clave universal, multiplicando capacidades comparativas y otorgando a la labor histórica la posibilidad de contribuir a la mencionada aspiración braudeliana de la historia total.

La CPR, puede ser entendidas como una matriz de convergencia del espacio físico y social donde se opera la interconexión de productos y bienes, ejerciendo entre sus partes un rol de diferenciación funcional, jerarquización de las relaciones de poder y articulación. Matriz que a su vez se encuentra conectada con otras redes de intercambio (Van Young, 1985). 
Asimismo, las CPR pueden ser observadas en su carácter de "unidad territorial emergente de la estructura espacial de la sociedad, que se convierte en una entidad establecida tanto y en cuanto sea reconocida en esferas de acción y la conciencia colectiva" (García Álvarez, 2006, p. 46). Este proceso, al que la Geografía Regional define como "institucionalización regional", implica el desarrollo de una forma conceptual y simbólica y el reconocimiento claro de su existencia, más allá de la obtención o no de un rango administrativo. Es entonces clave estudiar a las regiones en su perspectiva política cultural, en la faceta narrativa retórica o discursiva de la región. "Ciertamente las regiones son, en parte, relatos, narraciones en la medida que se cosifican, se hacen visibles y se comunican a la comunidad a través de determinados discursos" (García Álvarez, 2006).

Este aspecto ha sido de especial interés en distintas investigaciones que han dado lugar a publicaciones y acciones en materia de puesta en valor del reconocimiento identitario de Rosario y su región. En función de promover la integración de enfoques interdisciplinares, poner en valor el patrimonio documental portuario y constituir una plataforma interinstitucional de transferencia a las políticas públicas, se creó en 2016 el Núcleo de Historia de las Ciudades Portuarias Regionales (Núcleo CPR) en el marco del Nodo Instituto de Historia IDEHESI-CONICET. Se decidió avanzar a partir de la experiencia piloto en Rosario, y se propuso al Ente Administrador del Puerto de Rosario (ENAPRO) la instrumentación de un plan de preservación integral del patrimonio portuario de la institución.

\section{La hora metodológica sistemica y transdisciplinar}

Al analizar la historia de los archipiélagos ibéricos Guimerá Ravina aplicó un enfoque sistémico, afirmando que "los puertos y sistemas portuarios son entes complejos" que al ser contemplados en otro de entidad mayor (insular, regional, nacional y supranacional), pasaban a convertirse en forma automática en subsistema del anterior, conservando los elementos que lo definían. En todos los casos ofrecían una dimensión pasiva y activa. En esta última los puertos eran agentes de modernización y cambio, generando sus propios procesos, entrelazando a sus actores, produciendo modernización económica, cultural y social en distintos niveles, y recibiendo inversiones externas. Era esta dimensión activa la que caracterizaba a las ciudades portuarias porque era "germen de burguesía y movimiento obrero, colaborador en el proceso de mundialización de la ciencia y generador de una cultura marítima" señala (Guimerá Ravina, 2006:211).

Pierre Vilar advertía que todos los sectores de la realidad pasada, presente y futura son interdependientes y que por lo tanto el estudio del desarrollo de una problemática, como las que nos ocupa, la dinámica $\mathrm{CPR}$, que hace al desarrollo, implica necesariamente la realización de estudios comparados (Vilar, 1993). Al respecto, Braudel recordaba las palabras de su maestro Lucien Febvre, sobre la conveniencia de que los historiadores, "más allá de la práctica de una especialización legítima en el cultivo de su jardín se esforzaran en mantenerse al corriente de la labor del vecino para enriquecerse respecto del método y la interpretación de los hechos" (Braudel, 1968:40), con una finalidad social "vivir la historia" contribuyendo a un humanismo moderno, e invitaba a iniciar el diálogo con las ciencias humanas, primero con la geografía y luego con la sociología, rompiendo las fronteras entre especialistas.

La renovación de las corrientes historiográficas y la permeabilidad de las Ciencias Sociales al diálogo interdisciplinar permite deducir la existencia de un temperamento favorable a la apertura de campos integradores como el propuesto: el estudio histórico de la dinámica de las ciudades portuarias regionales (en adelante EHD-CPR). 
Ello implica la combinación de conocimientos científicos, orientados a lo social y a la transformación en el conocimiento político (Klein, 2004), la ausencia de un sistema portuario nacional federal e integrado capaz de alentar, fomentar y desarrollar estudios sobre su propia dinámica, el desconocimiento de las interfaces que afectan la calidad de vida de los ciudadanos, la dispersión de fuentes documentales, y la existencia de intereses que se benefician de las actuales asimetrías.

El estudio de la dinámica de las CPR es por lo tanto una apuesta a favorecer el estudio transdisciplinario, -reemplazando el sectorialismo metodológico por el sistemismo metodológico-, "recompensando el traspaso de límites en vez de castigarlo" (Bunge, 1999:487) y por ende contribuir al fortalecimiento de las Ciencias Sociales en su conjunto. "Rigor, apertura y tolerancia son las características fundamentales de la actitud y visión transdisciplinaria. El rigor en la argumentación que toma en cuenta toda la información disponible es la mejor barrera contra toda posible deriva. La apertura implica la aceptación de lo desconocido, lo inesperado y lo imprevisible. La tolerancia es el reconocimiento del derecho a las ideas y verdades opuestas a las nuestras", especifica el Artículo 14 de la Carta de Transversalidad. Congreso Internacional sobre Transdisciplinariedad, Portugal, 1994.

El EDH-CPR tiene una motivación transdisciplinar en función a los siguientes objetivos: 1 . Contribuir a la lúcida comprensión de las tensiones y desafíos del presente; 2 . Cooperar en la preservación de fuentes y patrimonio de las CPR en peligro; 3 . Aportar al desafío de la integración puerto, ciudad y región; 4 . Explicar procesos comunicacionales de la CPR; y 5. Favorecer la elaboración de protocolos para dar respuesta a la complejidad creciente de dicha dinámica.

En relación con estos puntos, el EDH-CPR se propone detectar, preservar y sistematizar la documentación histórica existente, -dentro de los recintos portuarios, y en diversos repositorios públicos y privados- a través del empleo de una ontología informática comunicacional que permita examinar determinadas constantes que en la historia han marcado su prosperidad, estancamiento y atraso de las áreas de influencia, como son su situación geográfica, la proximidad a los mercados, a los centros de producción o consumo o a nudos de comunicaciones importantes; la abundancia de espacio para las operaciones; la adaptación de las infraestructuras adecuadas a las características de los tráficos marítimos; la bondad de sus accesos terrestres y marítimos que permitan la conexión con las grandes rutas de transporte y garanticen la eficiencia intermodal; los bajos costes; la fiabilidad y seguridad en la operativa; el tipo de celeridad administrativa, tipo de equipamiento y sistemas de información. En resumen, a partir del reconocimiento de la naturaleza esencialmente competitiva de las CPR, de dominio de unas sobre otras, en el pasado, en el presente y en las perspectivas futuras, aspira a favorecer políticas para una gobernanza. Una aspiración a largo plazo y que se encuentra en una faz inicial, a través de un proyecto de cooperación para el estudio comparado entre la región de Bretaña (Francia) y los puertos cerealeros de ultramar de la Argentina.

\section{Las contribuciones disciplinares}

¿Puede la CPR ser entendida como una categoría científica particular, justificando aproximaciones conceptuales específicas, metodologías originales y esbozos de teorización? Cabría responder que al menos se puede admitir la singularidad del sistema como objeto específico versátil para el encuentro disciplinar. Por ejemplo, las CPR son de directa incumbencia de la geohistoria como ciencia que estudia la dinámica entre una sociedad del pasado y la estructura geográfica que la sustenta. Asimismo de la geografía portuaria, el estudiar el reconocimiento del origen-destino de sus flujos permite con 
claridad discriminarlos en: a) Terrestres (hinterlands), b) Marítimos (forelands) y c) Tipología de combinada (landbridge); y al observar las áreas de influencia (AIP) las divide en: a) Infraestructurales, es decir oferta, organización y sistema portuario; b) Locacionales, respecto a las grandes rutas oceánicas, a accidentes y unidades fisiográficas, y a la demanda; c) Económicas, demográficas y sociales; e) Dinámicas, la especificidad o diversificación de la economía dependiente del puerto en relación con su entorno próximo, y f) históricas que dan identidad a cada AIP. Asimismo ha clasificado los distintos tipos de puertos físicamente (natural o artificial), funcionalmente (pesquero, refugio, comerciales, de pasajeros, bases militares y deportivos), por titularidad y nivel de gestión (público o privado, y en el primer de los casos, depender de un gobierno central, autonómico, provincial o regional) y por servicios prestados (primera, segunda y tercera generación). Ha contribuido a la caracterización de las generaciones de puertos: Primera generación: Un puerto desligado del mercado en el que se asienta sin participar en la promoción y desarrollo de ese mercado. Segunda generación: Hinterland cautivo, y vinculados a una producción local de mucho volumen. Variadas interfaces. Enclavados en zonas de mediano desarrollo. Tercera generación: A lo anterior se suma que además logra una coordinación eficiente con el entorno económico y comercial, centro de transferencia. Cuarta, quinta y sexta generación son los del siglo XXI, que van incorporando el concepto de plataforma de comercio internacional con nuevas tecnologías de comunicación, con intermodalidad en el transporte y con una visión sistémica. Actúan de la manera más coordinada con otros puertos para el máximo rendimiento operativo con un sistema informatizado integrado.

El clásico esquema de Hoyle sobre los elementos de la geografía portuaria son de gran utilidad para el EDH-CPR al establecer aquellos aspectos teóricos, interdisciplinarios, temporales y espaciales que deben ser considerados en su análisis, en sus escalas locales, regionales, nacionales e internacionales (a los que en la actualidad correspondería agregar la global) y en los contextos (que son precisamente el área de mayor incumbencia histórica): economías avanzadas, de mediano y bajo desarrollo.

El análisis de la EDH-CPR comparte con las ciencias económicas, la logística, transportes y comunicación, así como el interés por procesos de "formación de excedentes, almacenamiento, transporte y distribución" (Liatis, 2015). La CPR interviene y puede determinar la corriente de circulación de productos tangibles e intangibles, entre ellos el circuito del conocimiento cultural. Son más propicios que otros a la innovación o a la transferencia de la innovación aplicada a la modernización institucional y al desarrollo económico. La capacidad transaccional de la ciudad se proyecta en su puerto, y, por lo tanto, dinamizar la ciudad fue dinamizar el puerto. El conocimiento de rol de las CPR en el relacionamiento de las fuerzas económicas y productivas representa un factor estratégico, no exento de tensiones. El conocimiento de ese proceso en su devenir histórico es relevante. Las CPR suelen ser matrices y gestoras de distintas áreas de influencia y de allí que los puertos son claves en la demarcación de los hinterland y foreland, actuando como "Hub", "Gateway", "Puerto seco", "Puerto ciudadano" y "Zona logística” (Rúa Costa, 2006:14).

Asimismo, el EDH-CPR no puede prescindir del conocimiento de la evolución del comercio internacional y de las Relaciones Internacionales, estrechamente vinculados entre sí al punto que pueden observarse patrones comunes en las CPR proteccionistas y librecambistas, con su correlato en el desarrollo de los hinterland y en los ritmos de determinado tipo de articulación entre el interior y el exterior (Martner Peyrelongue, 1999). Sin embargo, es erróneo interpretar a la actividad portuaria sólo en función del comercio exterior porque de ella depende la elaboración de estrategias para el desarrollo de su entorno, que a su vez incide en la calidad de vida de los habitantes de su hinterland. El puerto, en este sentido, no debe ser una puerta al atraso o a la dependencia, y por eso la historia enseña que ninguna ciudad puerto tiene garantizada su prosperidad 
por el sólo hecho de serlo. Quizás uno de los ejemplos más elocuentes fue lo sucedido con el puerto chileno de Punta Arenas y la economía de toda la región magallánica a consecuencia de la inauguración del Canal de Panamá, en 1914. De ser el más floreciente puerto en el extremo sur de América por el que pasaba todo el tráfico destinado a la costa sudoriental del Pacífico proveniente del estrecho de Magallanes, pasó a ser un puerto de recalada final de la misma corriente, con un hinterland y foreland mucho más restringido, perdiendo así su esplendor.

El estudio de la evolución histórica de las interfaces CPR puede de alguna manera explicar el presente y advertir su futuro.

Además, puede ayudar a observar las articulaciones pasadas en la estructuración y conservación de un orden social particular dentro de un marco cultural determinado, que da sentido al presente (Sánchez Calderón, 2013), y allí pueden trabajar mancomunadamente profesionales provenientes de la sociología, la filosofía, la psicología social, la etnología, la antropología y las ciencias de la educación. El referido estudio de las CPR comparte además el interés por el trasvasamiento de las elites portuarias, las inmigraciones y migraciones, la aceptación de alteridades, las identidades cosmopolitas y refractarias; la inclusión o exclusión; el surgimiento de conductas, actitudes y aptitudes; y la receptividad o rechazo de paradigmas internacionales del conocimiento. Teniendo en cuenta que cada CPR es ámbito de elaboración y aplicación de políticas públicas, de los proyectos de región, país, y nación, participa con las Ciencias Políticas del interés por entender los mecanismos de tensiones, consensos y disputas entre los actores individuales y colectivos por el control de los canales de relacionamientos (Ansaldi, 1994).

La CPR a través de la regulación de la comunicación replican escaladas simétricas o asimétricas proporcionales a su capacidad de ordenar e imponer relaciones de poder, jerarquías, identidades, discursos, regulaciones, derechos y deberes, prioridades, demandas, la percepción de lo público y privado con incidencia directa tanto en lo cotidiano como en dichos aspectos que hacen a lo esencial de la vida misma (Isolda Carranza, 2006).

La puesta en valor, preservación y difusión del patrimonio cultural urbano encuentra en el EDH-CPR una explicación inclusiva de aquellos aspectos intervinientes en la integración puerto-ciudad-región. Además, las instalaciones portuarias, en especial las que no se encuentran afectadas a la operatoria directa, son una cátedra a cielo abierto sobre los desafíos de la integración, y los intereses intervinientes, muchos ellos en disputas y las causas de la generalizada pérdida del patrimonio histórico cultural de los puertos.

La concreción del bello e imponente Complejo Cultural Parque de España, en Rosario, es interpretado como parte de una larga lucha de la ciudad por acceder a la zona ribereña, recuperando el vínculo con el entorno del río Paraná. Difícilmente se hubiera podido erigir a no ser por la dinámica de Rosario como CPR, en máxima interacción de los actores de sus interfaces con décadas de funcionamiento. En 1992 se inauguró la primera etapa y a partir de allí se inició un proceso de reutilización de cada vez más antiguas instalaciones del mismo tipo con la apertura y valorización de la rivera central en una gran extensión. Las visiones y las vivencias "de la ciudad" ya no serían la misma. El Centro Cultural Parque de España por su parte se convirtió en un ámbito de revalorización del Paraná, las islas, y las expresiones artísticas (Martín Prieto, 2011).

Desde el punto de vista epistemológico y de la historia de la ciencia, hay estudios que permiten seguir la evolución de artefactos de distinta índole construidos para la operatoria portuaria y el transporte regional. Las herramientas provistas por las humanidades digitales, especialmente para la preservación del patrimonio histórico portuario (Laubé, 2012), revelan su potencial didáctico formativo. 


\section{Propuesta de trabajo: corredores, actores y producción}

La dinámica histórica de relacionamiento conformada por las secuencias de interfaces entre un puerto con la ciudad y la región, y su incidencia en la integración y el desarrollo de los respectivos hinterland es objeto de análisis en sí misma. Dinámica que, por su complejidad, como ya se ha señalado, requiere de enfoques transdisciplinares, interdisciplinares e interinstitucionales, y dentro de la historiografía, un esfuerzo de integración de especialistas en diversas temáticas (económicas, sociales, políticas, y culturales), perspectivas, y escalas de análisis: local, regional, nacional e internacional.

El estudio comparado de la evolución de distintas CPR arroja disparidad en el desarrollo, posible de ser evaluado éste según el grado de interrelación de todas las fuerzas sobre las que ejerció un rol de mediación. De allí que el estudio del desarrollo de regiones surgidas a partir de una ciudad y su puerto debería previamente identificar las partes del sistema para evaluar en qué medida la combinación de estas contribuyó al desarrollo del conjunto. En la historia de Rosario he podido distinguir tres grandes áreas de interfaces que intervienen en el ritmo de la dinámica CPR: corredores o canales; actores de la transferencia; y la resultante de la mediación que retroalimentan y fortalecen el circuito.

Cuadro 1. Interfaces de la dinámica de las ciudades portuarias regionales. Fuente: Miguel Ángel De Marco (h)

\begin{tabular}{|c|c|c|}
\hline $\begin{array}{l}\text { Corredores } \\
\text { De flujos a partir del puerto }\end{array}$ & $\begin{array}{l}\text { Actores } \\
\text { "Del movimiento", entre el puerto } \\
\text { ciudad región y foreland }\end{array}$ & Artefactos \\
\hline $\begin{array}{l}\text { Por sentido: } \\
\text { *Foreland-Hinterland } \\
\text { *Hinterland-Foreland } \\
\text { Por inserción: } \\
\text { *Económica. } \\
\text { *Política. } \\
\text { *Militar. } \\
\text { Por intensidad: } \\
\text { *Áreas de transición. } \\
\text { *Áreas críticas. } \\
\text { *Áreas consensuadas. } \\
\text { Por el alcance: } \\
\text { *Locales } \\
\text { *Regional próximo } \\
\text { *Regional mediano } \\
\text { *Regional lejano } \\
\text { *Foreland } \\
\text { Por fases históricas: } \\
\text { *Enclave portuario } \\
\text { *Ciudad Puerto } \\
\text { *Ciudad Portuaria } \\
\text { *Ciudad Puerto Industrial } \\
\text { *Desplazamiento de la ciudad } \\
\text { *Refuncionalización espacios } \\
\text { portuarios en desuso. }\end{array}$ & $\begin{array}{l}\text { Por escala: } \\
\text { *De la operativa portuaria (dentro y } \\
\text { fuera del recinto). } \\
\text { *Del hinterland epicéntrico (la ciudad, } \\
\text { cordones urbanos y la región próxima). } \\
\text { *Del hinterland inmediato (región } \\
\text { provincial e interprovincial). } \\
\text { *Del hinterland mediato (nacional). } \\
\text { Del hinterland fraterno (países } \\
\text { limítrofes). } \\
\text { *Del foreland (en relación con lo } \\
\text { internacional). } \\
\text { Por áreas de relación: } \\
\text { *Operatoria. } \\
\text { *Comunidad portuaria. } \\
\text { *Cadena logística. } \\
\text { *Mercado y comercialización. } \\
\text { *Producción. } \\
\text { *Empresas. } \\
\text { *Transportes y accesibilidad. } \\
\text { *Gobernanza y gestión pública. } \\
\text { *Comunicación social. } \\
\text { *Espacios de uso social. } \\
\text { *Formación de recursos. } \\
\text { *Preservación de la identidad } \\
\text { arquitectónica y portuarias }\end{array}$ & $\begin{array}{l}\text { *Construcción } \\
\text { identitaria. } \\
\text { *Transferencia del } \\
\text { conocimiento y } \\
\text { formación de recursos. } \\
\text { *Políticas públicas. } \\
\text { *Desarrollo. } \\
\text { *Integración. } \\
\text { *Innovación. } \\
\text { *Relaciones } \\
\text { interestatales y } \\
\text { organizaciones, } \\
\text { locales, nacionales e } \\
\text { internacionales. } \\
\text { *Mecanismos de } \\
\text { aceptación o rechazo. } \\
\text { *Producción } \\
\text { tecnológica. } \\
\text { *Canales de } \\
\text { comunicación. } \\
\text { *Trayectos de vida. } \\
\text { *Paisajes portuarios. } \\
\text { *Patrimonio cultural. }\end{array}$ \\
\hline
\end{tabular}

\section{Corredores de las interfaces}

El concepto "interfaz" comenzó a ser utilizado profusamente a partir de su utilización en el universo digital pero ya ha sido incorporado por distintas disciplinas. Desde el diseño y la comunicación visual es considerado como "un dispositivo diseñado y usado para facilitar la relación de sistemas" a manera de "punta de iceberg de un sistema complejo de ag(entes) interdependientes: infraestructuras, códigos, datos, usos, leyes, corporaciones, sonidos, espacios, comportamientos, objetos, protocolos, botones, 
tiempos, afectos, efectos, defectos...", "que responde en tiempo y espacio a un contexto cultural, a una lógica del sistema y a una ideología no siempre visible pero si presente" (Figuerola y Marzo, 2016:48).

Un valioso aporte proveniente del campo de la ciencia de la información y las humanidades digitales define a la interfaz como la instancia mediadora de la comunicación (Laitano, 2015), con la capacidad para hacer comprensible un sistema.

En el lenguaje técnico, urbanístico y arquitectónico, la interfaz es una zona que determina el empalme o conexión de patrones dentro de un sistema, dándole consistencia interna y/o conectividad externa, o la comunicación de un sistema sobre otro.

Así pues, el sistema CPR es una interfaz con conexiones internas funcionales a una dinámica de integración de tres sistemas complejos y multifuncionales: Ciudad-PuertoRegión, y una de las tareas de aquellos interesados en su estudio es por lo tanto detectarlas para su posterior conocimiento y diagnóstico.

Si se analiza a la CPR ya no en el aspecto teórico sino en la práctica de relación entre puerto y ciudad, se ha reconocido la existencia de un área de interfaces entre los espacios terrestres y marítimos en las instalaciones portuarias y en la infraestructura para el acceso terrestre y marítimo. Interfaces que se han modificado junto a la evolución de los puertos que siguiendo a la tipología Anyport (Bird, 1977) comprenden las siguientes etapas: Asentamiento, expansión y especialización, como una adaptación a las innovaciones de la economía internacional y el transporte marítimo mundial. En la actualidad, y de acuerdo a los intercambios de experiencias en los encuentros de la Asociación Internacional de Villa y Puertos (AIVP), las interfaces de la ciudad puerto son "territorios complejos" no exentos de tensiones, porque alli "se cristalizan los desafíos de la competencia y de la complementariedad urbana y portuaria ante recursos de espacio que muchas veces son restringidos", y tienen un alcance territorial que se trasunta a la región contigua. Las áreas de interfaces generalmente son "espacios de transición", entre la ciudad, el puerto y la región, y por ende es ahí donde se producen negociaciones ante problemas como la congestión, el transporte, la accesibilidad, la revitalización de los frentes costeros y la forma de como preservar la identidad arquitectónica y portuaria” (Guía AIVP “Hacer la ciudad con el puerto”, 2015).

Las ciudades portuarias son un área de intenso dinamismo, y de elevada densidad y multiplicidad de interfaces, y mucho más cuando las mismas intervienen sobre la región contigua, afectando la calidad y sostenibilidad del desarrollo del conjunto. El EHD-CPR, por lo tanto, puede explicar los contextos, causas y consecuencias de los conflictos, crisis, acuerdos y consensos que modelaron el complejo CPR y lo condicionan en la actualidad.

\section{Actores}

Si se entiende a la interface como "el conjunto de comunicaciones concretas, los intercambios perceptibles entre polos que guardan sentido" (Laitano, 2015:113-114), se hace imprescindible conocer a los actores grupales e individuales que intervinieron en la configuración relacional de las CPR, en su espacio urbano, en su hinterland y foreland, desempeñando diversos roles de acuerdo con las particularidades de cada sistema CPR (función, flujos, conocimientos, tecnología, inserción en el contexto internacional, entre otros aspectos).

Una trama que obliga a ponderar la participación no sólo de los integrantes de la "comunidad portuaria", los agentes de la administración y los responsables de la actividad logística sino también a los que hicieron posible una dinámica de interacción puerto-ciudad-región fuera de la terminal. 
En primer término, sería conveniente identificar los actores históricos de una ciudad portuaria regional, tipificarlos, establecer sus funciones y valorar sus desempeños en la secuencia de interfaces, los que por escala de relacionamiento pueden dividirse en: actores del área operativa de la actividad portuaria (dentro y fuera del recinto portuario); del hinterland epicéntrico (la ciudad, cordones urbanos y la región próxima); del hinterland inmediato (región provincial e interprovincial); del hinterland mediato (nacional); del hinterland fraterno (países limítrofes), y del foreland (en relación con lo internacional).

De acuerdo el perfil disciplinar se podría inferir subdividir a estos actores según el nivel de decisión: individual, grupal (elites, asociaciones, entidades de bien público, de la producción, etc.), civiles (tradicionales, no tradicionales o intermedios) o estatales (en todas sus órbitas y niveles). Libourel en su estudio sobre los corredores del Mediterráneo, siguiendo a la definición del espacio geográfico de Roger Brunet, sostiene que dentro de los actores institucionales se encontrarían las instancias comunitarias europeas, estados, operadores estatales, puertos autónomos, comunidades autónomas, provincias y municipios. En tanto divide a los actores no institucionales en individuales: miembros de la sociedad civil, empresas, operadores de planeamiento y clústers; y en colectivos: asociaciones sectoriales, asociaciones territoriales, organizaciones representativas, think-tanks y grupos de presión. Unos y otros ejercen presiones entre sí (Libourel, 2016).

Los actores portuarios tienen historicidad y por lo tanto convendría comprenderlos en la evolución de contextos de poder, ciclos económicos, conflictos y acuerdos.

\section{Producido}

Un tercer aspecto a considerar en el EHD-CPR es la existencia de producción resultante de la secuencia de las dos interfaces señaladas, corredores y actores: aquello producido, bienes tangibles e intangibles para la perdurabilidad de la dinámica que integra al sistema. Eso "producido" se puede asociar a un "artefacto", tal como se presentan en la propuesta modélica de Laubé-Rohou, como la resultante de los actores y la generación de saberes. Desde la psicología instrumental un "artefacto" es aquello "desarrollado para encajar en actividades finalizadas, que implementa una solución a un problema o a una clase de problemas socialmente planteados" (Laitano, 2015:68) y por ende, puede ser material o simbólico. El "artefacto", en relación con el EHD-CPR, podría ser entendido como la resultante de la relación entre la CPR con sus actores y los integrantes del NH-CPR han puesto su atención en los diversos "corredores" en que dichos artefactos fueron detectados: a) Construcción identitaria; b) Transferencia del conocimiento y formación de recursos; c) Políticas públicas; d) Desarrollo; e) Integración; f) Relaciones interestatales y organizaciones internacionales; g) Trayectos de vida; h) Paisajes portuarios; e i) Patrimonio cultural.

Este último punto -que comprende la preservación, producción de formas de intervención y difusión del patrimonio histórico-cultural de las ciudades portuarias regionales-, reviste un carácter apremiante y por eso se impulsó la adopción de una estrategia y metodología común para la puesta en valor de las fuentes documentales.

Las ciudades portuarias de la Argentina enfrentan similares desafíos como consecuencia de la ausencia de políticas específicas para la preservación del patrimonio histórico portuario nacional, provincial y local, entre ellos, la dispersión de las fuentes y los escasos recursos destinados a su salvaguarda. Un obstáculo que también enfrentan los interesados en el conocimiento de la relación puerto y ciudad en la actualidad. Geógrafos, urbanistas, economistas, ambientalistas, etc. han advertido sobre la ausencia de datos que permitan conocer y evaluar con mayor exactitud la interdependencia 
puerto ciudad, los hinterland de cada puerto, la proporción de sus flujos, el impacto de los cambios de operatorias y relocalizaciones portuarias en las desigualdades sociales, etc. y en el terreno del análisis científico, definir incluso si se está ante una modalidad de ciudad puerto de una ciudad portuaria. En cambio, se suele manejar información parcial que debilita la posibilidad de efectuar estudios comparados. (Ducruet, 2011:9).

Al ser la salvaguarda de documentación histórica una problemática común que afecta además del patrimonio histórico la labor investigativa de especialistas en diversas temáticas historiográficas y disciplinas sociales, desde el NH-CPR se ha trabajado en red pensando en estrategias generales para lo cual se han iniciado acciones ante autoridades portuarias, con distinto resultados.

Sobre el relevamiento inicial de la documentación existente en los puertos de Necochea, Mar del Plata y Rosario por el profesor doctor Bruno Rohou, investigador del Centrè Francois Vieté de la Universidad de Bretagne Occidentale, (CFV-UBO), se decidió promover el rescate de dichos fondos. En el caso Rosario, de la documentación "rescatada" se desprenden elementos que permiten identificar a múltiples actores de la dinámica ciudad-puerto y elaborar un "nomenclador" provisorio de los mismos, conducente a la comprensión de secuencias de interfaces.

El CFV-UBO, bajo la dirección de los investigadores Silvayne Laubé y Serge Gerlatti, se ha especializado en humanidades digitales aplicadas a la historia de los puertos y a la historia de la tecnología. A partir del modelo genérico Anyport (Bird, J. 1963), que permite periodizar varios puertos estudiando la evolución en el tiempo de las infraestructuras portuarias, Laubé desarrolló el modelo Any-Artefact, que considera que una actividad humana implica uno o varios actores (individuo o grupo social), un artefacto (o sistema de artefactos) y los conocimientos específicos a esta actividad. Rohou contó con la colaboración de integrantes del NH-CPR, y ha recogido información conducente a su tesis doctoral con el objetivo determinar las grandes fases de evolución y los momentos de ruptura entre fases, respecto a los puertos considerados (Rohou, Gerlatti y Laubé, 2017).

En la actualidad se sostiene con ese centro de investigación francés una estrecha relación que ha permitido la realización de coloquios, jornadas, encuentros e investigaciones conjuntas, y la ejecución del proyecto Ecosur, tal como se mencionó. De contar con los recursos necesarios no se descarta aplicar dicho modelo interpretativo al caso argentino en lo relacionado con la sistematización de los procesos de interfaces de las $\mathrm{CPR}$, habiéndose iniciado, en una primera etapa, la ontología vinculada a los actores institucionales. ${ }^{4}$

\section{Consideraciones finales}

Las nociones de "ciudad", "puerto", "regional", CPR, que atraviesan puentes teóricos entre neoinstitucionalismo económico, geohistoria, urbanismo e historia regional, además de subdisciplinas adyacentes, es una invitación al análisis de una dinámica histórica de relacionamiento conformada por las secuencias de interfaces, identificando actores, saberes y producciones bajo el prisma de diversos campos de conocimiento 
científico. Dinámica que ha incidido, y lo sigue haciendo, en la gobernanza, el desarrollo sustentable y en la preservación del patrimonio histórico documental de los puertos. La puesta en valor, su rescate y su digitalización a los efectos de dotar de mayor flexibilidad a su gestión, adquiere en la Argentina un carácter apremiante, tal como venimos enfatizando, producto de la propia trayectoria investigativa, de acciones de concientización científica en ámbitos académicos y universitarios y particularmente del análisis comparativo al que se ha accedido mediante el trabajo cooperativo de investigadores de distinta procedencia y formación disciplinar. El entrecruzamiento de estos saberes y prácticas con los ámbitos de planeamiento de políticas públicas asume la urgente necesidad de transferir esos conocimientos teóricos y prácticos a los efectos de lograr una real incidencia en la gestión y ejecución de acciones para preservar el patrimonio portuario regional. 


\section{Q Bibliografía}

» Ansaldi, W. (1994). La interferencia está en el canal. Mediaciones políticas (partidarias y corporativas) en la construcción de la democracia argentina. Boletín americanista, XXXIV(44), 7-24.

» Aoki, M. (1996). Towards a Comparative Institutional Analysis: motivations and some tentative theorizing. The Japanese Economic Review, 47(1), 1.19.

»Areces, N.R. (1999). Regiones y fronteras, apuntes desde la historia. Revista Andes, 10, 19-31.

»Arias Bucciarelli, M. (2009). La Patagonia argentina como Territorio Nacional. Perspectivas de análisis, Octavo Congreso de Historia Social y Política de la Patagonia Argentino-Chilena, Chubut, Argentina.

»Assauduriam, C. (1983). El sistema de la economía colonial. El mercado interior, regiones, y espacio económico. México. Instituto de Estudios Peruanos.

" Bandieri, S. (2001). La posibilidad operativa de la Construcción Histórica Regional o cómo contribuir a una historia nacional más complejizada. En S. Fernández y G. Dalla Corte (Eds.), Lugares para la Historia, Espacio, Historia Regional e Historia Local en los Estudios Contemporáneos, 91-119.

»Bird, J. (1977). Centrality and Cities. Londres, Routledge Direct Editions.

» Bird, J. (1967) Seaports in the European Conmunity, Geograph Journal, 33, 302327. Londres.

» Bonil, J., Sanmartí, N., Tomás, C. y Pujol, R.M. (2004). Un nuevo marco para orientar respuestas a las dinámicas sociales: el paradigma de la complejidad. Investigación en la escuela, 53, 1-20.

》Braudel, F. (1970). La historia y las ciencias sociales Alianza Editorial, Madrid.

» Braudel, F. (1977). La dinámica del capitalismo, Fondo de Cultura Económica, Buenos Aires.

》 Braudel, F. (1993). La Identidad de Francia, I, Gedisa Editorial, Barcelona.

"Broeze, F. (1987). Gateways of Asia. (1987), Port Cities of Asia in the 13th-2oth Centuries, London-New York.

»Caro, J.E. y Vidal Ortega, A. (2009). Ciudades portuarias de la Cuenca del Caribe, historia, cultura, economía y sociedad, Universidad del MagdalenaUniversidad del Norte, Santa Marta.

»Carta de Transversalidad (1994). Congreso Internacional sobre Transdisciplinariedad, Convento da Arrábida, Portugal.

"Carranza, I. (2006). El relato y el orden social, en A. Parini y A. Zorrilla (Eds.), Lengua y Sociedad, 137-153, Buenos Aires, Áncora.

" Castejón, R. (1999). Análisis portuario en la Geografía española. Revista de Geografía, 24, 1-38.

»Ducruet, C. (2011), The port city in multidisciplinary analysis. En The port city in the XXIst century: New challenges in the relationship between port and city, RETE, 32-48.

»Ducruet, C., Cuyala, S., El Hosni, A. (2018). Maritime networks as systems of cities: The long-term interdependencies between global shipping flows and 
urban development (1890-2010). Journal of Transport Geography, 66, 340-355.

»Ducruet, C., Notteboom, T., y De Langen, P. (2009). Revisiting inter-port relationships under the New Economic Geography research framework. En Transport and mobility, 11-27.

»Fernández, S. (2008), El revés de la trama: contexto y problemas de la historia regional y local, en S. Bandieri, G. Blanco y M. Blanco, (Coords.) Las Escalas de la Historia comparada, 2, 233-247.

» Fujita, M. y Krugman, P. (2004). La nueva geografía económica, pasado, presente y futuro, Investigaciones Regionales, 4, 177-206.

» Gallego Martínez, D. (2014). Las distintas caras de la economía institucional, Documentos de Trabajo, 1412, 1-45.

" García Álvarez, J. (2006). Geografía regional, en A. Lindón y D. Hiernaux (Eds.), Tratado de Geografía Humana, 25-70.

" García, A. y Rofman, A. (2013). Poder y espacio. Hacia una revisión teórica de la cuestión regional en Argentina. Revistas Problemas del Desarrollo, 175(44), 101124 .

» Guimerá Ravina, A. y Monge, F. (1999). La Habana, puerto colonial (Siglos XVIII y $X I X)$. Fundación Portuaria, Madrid.

" Guimerá Ravina, A. (2003) El vino y los puertos de la Bahía de Cádiz, siglos XVIII y XIX, en L. Ribot García, L. de Rosa (Dir.), Naves, puertos e itinerarios marítimos en la Época Moderna, 44-64.

" Greif, A. Historical and Comparative Institutional Analysis, The American Economic Review, 88(2), 80-84.

» Hayuth, Y. (1989). The Dynamics and dimensions of Port-City Interrelationships. Geoforum, 20(20), 427-428.

" Hoyle, B.S., Hilling, D. (1989). Seaports Systems and Spatial Change. Technology, Industry and Development Strategies, Chichester.

» Jumar, F. (2012). El complejo portuario rioplatense durante el Antiguo Régimen. Problemas y posibilidades explicativas, IV Jornadas Interdisciplinarias de Estudios Portuarios, La Plata.

" Kraselsky, J. (2012). Las corporaciones mercantiles en Río de la Plata y sus estrategias colectivas de negociación: la centralización corporativa, f. s. XVIII y ppios. s. XIX, en IV Jornadas Interdisciplinarias de Estudios Portuarios, La Plata.

"Law, J. (2000). Actor Network Theory and Material Semiotics, Lancaster University.

"Laitano, M.I. (2015). Le modèle trifocal: une approche communicationnelle des interfaces numériques: Contributions à la conception d'interfaces accessibles. Sciences de l'information et de la communication. Paris

" Liatis, R. y Fiadone, R, y otros. (2015). Historia de la logística en la Argentina. Buenos Aires.

" Libourel, E. (2016). El Corredor Mediterráneo: Desencuentro político y territorial, Valencia.

» Mata de López, S. (2003), Historia local, historia regional e historia nacional. ¿Una historia posible?, en Revista Escuela de Historia, 1(2).

» Martínez Figuerola, T. y Marzo, J.L. (2016). Interface Politics. 1st International Conference 2016. Barcelona. 
" Martner Peyrelongue, C. (1999). El puerto y la vinculación entre lo local y lo global, Revista Eure, 25(75), 103-120.

»Monge, F. (2000). Ciudades portuarias y dinámica sociocultural, Los puertos españoles: historia y futuro (siglos XVI-XX), 89-103.

» Notteboom, T. y Rodrigue J. P. (2005). Regionalización portuaria: hacia una nueva fase en el desarrollo portuario, Política y gestión marítima, 32(3), 297-313.

»Pesquera, M.A. y Ruiz, J.R. (1996). Estrategias de desarrollo sostenible para las ciudades puertos, Nueva York-Ginebra.

»Price, J. (1974). Economic Function and the Growth American Port Towns in the Eighteenth Century, Perspectives in American History, 8. 123-186.

»Prieto, M. y Silvestri, G. (2011). Paraná Ra'anga. Un viaje filosófico, Centre Cultural

»Centro Cultural Parque de España, Revista Altaïr S.A., Rosario-Barcelona.

» Regalsky, A. M. (1999). Exportaciones de capital y grupos inversores: las inversiones francesas en la Argentina, 1880-1914. Anuario de Estudios Americanos, 56 (2). 553-580.

»Rohou, B. Gerlatti, S. y Laube, S. (2017). Periodizar y comparar la evolución de los puertos: Interés cruzado de las humanidades digitales en el enfoque de la historia de la ciencia y de la técnica aplicado a los puertos de Brest (Francia), Mar del Plata y Rosario (Argentina), XVI Jornadas Interescuelas de Departamentos de Historias, Mar del Plata.

"Rúa Costa, C. (2006). Los puertos en el transporte marítimo, Instituto de Organización y Control de Sistemas Industriales, Cataluña.

"Rueda, G., Sazatornil, L. y Delgado, C. (2008). Las principales ciudades portuarias españolas en el siglo XIX, IX Congreso Internacional de la Asociación Española de Historia Económica, Murcia.

» Sánchez Calderón, F.V. (2013). Hacia la multiplicidad del espacio en la historia. Relaciones entre el cambio social y los cambios en la disciplina en las últimas cuatro décadas. Revista de Estudios Sociales 47, 39-50.

»Suárez Bosa, M., Jiménez González, J. J. y Castillo Hidalgo, D. (2011). Puerto, Empresas y Ciudad: una aproximación histórica, al caso de Las Palmas de Gran Canaria, Revista de Historia Industrial, 46, 73-96.

»Solano, S. P. (2019). Del espacio portuario a la ciudad portuaria los puertos del Caribe colombiano como espacios polifuncionales en el siglo XIX, en Varia Historia, 26 (44). 573-589.

»Tobal Conesa, C. (1997). Nuevas perspectivas en la geografía portuaria: las relaciones puerto-ciudad, Revista Bibliográfica de Geografía y Ciencias Sociales 24, https://www.raco.cat/index.php/Biblio3w/article/view/65457

» Thompson Klein, J.T. (2004). Transdisciplinariedad: Discurso, Integración y Evaluación, en Transdisciplinariedad y Complejidad en el Análisis Social, Gestión de las Transformaciones Sociales MOST, 70, 30-40.

»Van Young, E. (1987). Haciendo Historia Regional: Consideraciones metodológicas y teóricas, en Anuario IEHS, 2, 255-281.

»Vilar, P. (1993). Crecimiento y Desarrollo. Planeta/Agostini, Barcelona.

»Von Bertalanffy, L. (1976). General System theory: Foundations, Development, Applications, George Brazilliers, New York. 


\section{Miguel Ángel De Marco (h) / migueldemarco@conicet.gov.ar}

Doctor en Historia. Investigador del Instituto de Estudios Históricos, Económicos, Sociales e Internacionales (IDEHESI) - CONICET, Nodo IH Rosario. Director del Núcleo de las Ciudades Portuarias Regionales, Argentina. Docente titular de la Maestría de Historia Económica y las Políticas Públicas en Argentina, de la Facultad de Ciencias Económicas de la Universidad de Buenos Aires. Docente titular del Seminario Historia de los Puertos y del Desarrollo Regional en la Carrera de Doctorado en Historia en la Facultad de Historia, Turismo y Geografía, de la Universidad del Salvador. Miembro de Número de la Academia Nacional de la Historia. 